\title{
Ultrasound influence on behavior of disordered dislocation sys- tems in a crystal with non-equilibrium grain boundaries
}

\author{
D.V. Bachurin ${ }^{1,2}$, R.T. Murzaev ${ }^{1, \dagger}$ J.A. Baimova ${ }^{3}$, A.A. Samigullina ${ }^{1}$, K.A. Krylova ${ }^{1}$ \\ ${ }^{\dagger}$ mur611@mail.ru \\ ${ }^{1}$ Institute for Metals Superplasticity Problems RAS, Khalturin St. 39, 450001, Ufa, Russia \\ ${ }^{2}$ Institute for Applied Materials, Karlsruhe Institute of Technology, Hermann-von-Helmholtz-Platz 1, 76344, \\ Eggenstein-Leopoldshafen, Germany \\ ${ }^{3}$ M.N. Miheev Institute of Metal Physics of Ural Branch of RAS, Kovalyevskoy St. 18, 620990, Ekaterinburg, Russia
}

\begin{abstract}
This paper presents a numerical simulation of behavior of disordered dislocation systems under ultrasound influence in a model grain of two-dimensional polycrystal containing non-equilibrium grain boundaries. Non-equilibrium state is modeled via the stress field of wedge junction disclination quadrupole, which is a characteristic element of non-equilibrium structure of ultra-fine grained materials obtained by severe plastic deformation methods. It is assumed that the length of a standing sound wave is much larger than oscillation amplitude of the dislocations, and the stress amplitude is below the dynamic yield stress. Dislocation positions and the sign of their Burgers vector in the initial configuration are set randomly. All dislocations belong to the same slip system, but locate on different (parallel) glide planes. Qualitatively different behavior of the dislocation structures in contrast to the infinite crystal was found. Namely, no dislocation walls with alternating sign of the Burgers vector was found. Instead of this, if the number of dislocations is small, practically all dislocations move from the grain into the boundaries. Formation of a substructure consisting of dislocation walls, dipoles and multipoles occurs at higher dislocation densities. More pronounced "cleaning" of the grain from the dislocations takes place at increased amplitudes of ultrasonic influence. For quantitative assessment of relaxation of dislocation system, the trace of the internal stress tensor is calculated. The presence of two (fast and slow) relaxation stages was revealed. It is shown that the level of internal stresses, associated with the restructuring of disordered dislocation structure, can be reduced up to $20 \%$.
\end{abstract}

Keywords: disordered dislocation systems, dislocation dynamics, disclination quadrupole, ultrasound, ultrasonic treatment.

\section{Влияние ультразвука на поведение неупорядоченных дисло- кационных систем в кристалле с неравновесными границами зерен}

\author{
Бачурин Д.В. ${ }^{1,2}$, Мурзаев Р.Т. ${ }^{1, \dagger}$, Баимова Ю.А. ${ }^{3}$, Самигуллина А.А. ${ }^{1}$, Крылова К.А. ${ }^{1}$ \\ ${ }^{\dagger}$ mur611@mail.ru
}

\footnotetext{
${ }^{1}$ Институт проблем сверхпластичности металлов РАН, ул. Халтурина 39, 450001, Уфа, Россия

${ }^{2}$ Institute for Applied Materials, Karlsruhe Institute of Technology, Hermann-von-Helmholtz-Platz 1, 76344, Eggenstein-Leopoldshafen, Germany

${ }^{3}$ Институт физики металлов имени М.Н. Михеева УрО РАН, ул. Ковалевской 18, 620990, Екатеринбург, Россия
}

В работе проводится численное моделирование поведения неупорядоченных дислокационных систем под воздействием ультразвука в зерне модельного двумерного поликристалла, содержащего неравновесные границы зерен. Неравновесносное состояние границ моделируется при помощи поля напряжений квадруполя клиновых стыковых дисклинаций, который является характерным элементом неравновесной структуры ультрамелкозернистых материалов, полученных при помощи методов интенсивной пластической деформации. Полагается, что длина стоячей звуковой волны намного превосходит амплитуды колебаний дислокаций, а амплитуда напряжений ниже динамического предела текучести. Положения дислокаций и знаки их вектора Бюргерса в исходной конфигурации задаются случайным образом. Все дислокации принадлежат к одной системе скольжения, но находятся на разных (параллельных) плоскостях скольжения. Обнаружено качественно иное поведение дислокационных структур в отличие от слу- 
чая бесконечного кристалла. А именно, образования дислокационных стенок, чередующихся по знаку вектора Бюргерса, не происходит. Вместо этого, если число дислокаций системы невелико, то практически все они из тела зерна перемещаются в границы зерен, в то время как при более высоких плотностях образуется субструктура, состоящая из дислокационных стенок, диполей и мультиполей. При увеличении амплитуды ультразвукового воздействия происходит более тщательная «очистка» зерна от дислокаций. Для количественной оценки релаксации дислокационной системы рассчитывается сумма диагональных компонент тензора внутренних напряжений. Выявлено наличие двух (быстрой и медленной) стадий релаксации. Показывается, что уровень внутренних напряжений, связанный с перестройкой неупорядоченной дислокационной структуры, может уменьшаться до $20 \%$.

Ключевые слова: неупорядоченные дислокационные системы, динамика дислокаций, дисклинационный квадруполь, ультразвук, ультразвуковая обработка.

\section{1. Введение}

Ультразвуковая обработка может вызывать существенные изменения как микроструктуры материалов, так и значительно влиять на поведение дефектов в кристаллической решетке, что, в свою очередь, выражается в изменении свойств поликристалла [1-3]. Использование высоких амплитуд ультразвукового воздействия приводит к интенсивной генерации дислокаций и формированию ячеистой структуры [3,4], упрочнению [2], а также к зарождению трещин и последующему разрушению [5]. В то время как при умеренных амплитудах, ниже динамического предела текучести, наблюдается релаксация внутренних напряжений, связанная с перераспределением дислокаций и формированием более равновесной структуры [6].

Сочетание предварительной пластической деформации с ультразвукой обработкой позволяет повысить термическую стабильность микроструктуры ультрамелкозернистых материалов, а также увеличить характеристики пластичности и предела прочности $[7,8]$. Однако, для полного понимания процессов релаксации дислокационной структуры необходимо обратиться за помощью к компьютерному моделированию, являющемуся наиболее удобным способом изучения динамики дислокаций.

До настоящего времени моделирование воздействия ультразвука на неупорядоченные ансамбли скользящих дислокаций проводилось в основном в бесконечном монокристалле [9-12], исключая влияние границ зерен, которые в неравновесном состоянии могут создавать дальнодействующие поля напряжений. Выяснение механизмов релаксации и дислокационных перестроек в поликристаллах с дефектной структурой исключительно важно для понимания процесса воздействия ультразвука на структуру объемных наноматериалов.

Целью настоящей работы является численное моделирование поведения неупорядоченных дислокационных систем под действием ультразвуковой стоячей волны. Исследование проводится при помощи дискретно-дислокационного подхода в отдельно взятом зерне поликристалла с учетом неравновесного состояния границ зерен.

\section{2. Модель}

Пусть на некоторую систему, состоящую из $n$ краевых дислокаций, действует внешнее знакопеременное поле напряжений $\tau(t)$. При этом будем полагать, что $\tau(t)$ од- нородно в пределах области движения дислокаций, то есть, длина стоячей звуковой волны намного превосходит возможные амплитуды движения дислокаций; амплитуда знакопеременных напряжений ниже динамического предела текучести, и поэтому никакой генерации дислокаций не происходит; скорость скольжения дислокаций мала по сравнению со скоростью звука в среде. Пренебрежем влиянием кристаллографической ориентации образца на динамику дислокаций, а также влиянием разного рода примесных атомов и других дефектов. Будем рассматривать только скольжение дислокаций, полностью пренебрегая переползанием.

Пусть модельный двумерный поликристалл состоит из зерен квадратной формы. Рассмотрим одно зерно этого поликристалла. Пусть границы этого зерна содержат мезодефект, поле напряжений которого описывается с помощью квадруполя клиновых стыковых дисклинаций. Такой мезодефект может образоваться при пластической деформации поликристалла [13] и является характерным элементом неравновесной структуры ультрамелкозернистых материалов, полученных методами интенсивной пластической деформацией [14].

Обозначим через $x_{i}$ смещения $i$-ой дислокации из положения равновесия, а через $\tau_{i j}-$ сдвиговое напряжение, создаваемое $i$-ой краевой дислокацией в месте расположения $j$-ой. Тогда система уравнений движения для $i$-й дислокации системы может быть записана в виде:

$$
\frac{d x_{i}}{d t}=B\left(S_{i} \cdot \tau(t)+\sum_{\substack{j=1 \\ j \neq i}}^{n} \tau_{j i}\left(x_{i}-x_{j}, y_{i}-y_{j}\right)+\tau_{x y}^{q u a d}(x, y)\right)^{m} .
$$

Выражение, стоящее в скобках, представляет собой суммарное сдвиговое напряжение в плоскости скольжения дислокации в направлении вектора Бюргерса: первое слагаемое связано с внешним ультразвуковым воздействием [15]; второе - суммарное сдвиговое напряжение, действующее на $i$-ю дислокацию со стороны всех остальных дислокаций системы [16]; третье - сдвиговые напряжения, вызванные наличием дисклинационного квадруполя [17]. Здесь $B-$ коэффициент подвижности дислокаций; $m$ - показатель, который при небольших сдвиговых напряжениях равен нескольким единицам; $S_{i}= \pm 1$ в зависимости от знака вектора Бюргерса дислокации; $n$ - общее число дислокаций; $\tau(t)-$ внешнее напряжение, вызванное действием ультразвука, которое изменяется по синусоидальному закону $\tau(t)=\tau_{0} \sin \omega t$, где $\tau_{0}$ и $\omega-$ амплитуда и частота соответственно. Сдвиг по фазе положен равным нулю, что соответствует условию существования стоячей волны. Более подробное 
описание вывода выражения (1) приведено в наших предыдущих публикациях $[18,19]$.

Уравнения системы (1) решались численно с помощью метода Рунге-Кутта четвертого порядка. Показатель степени напряжения был выбран равным $m=3$. Следует отметить, что величина $m$ определяет степень нелинейности выражения (1) и влияет только на скорость движения дислокаций под действием ультразвука, но не на их взаимные перестройки, и поэтому в настоящей работе ее выбор диктовался лишь соображениями удобства расчетов. Шаг интегрирования: $d t=2 \pi \cdot 0.0001 / \omega$. Считалось, что в начальный момент времени все дислокации имеют нулевые значения скорости. Для расчета использовались следующие значения параметров: $B=10^{-5}$ Па.c; $b=2.482 \AA ; \mu=82$ ГПа; $v=0.29$; мощность дисклинаций $\Omega=1-2^{\circ} ; \tau_{0}<E / 2$, где $E$ модуль Юнга.

Исходное распределение дислокаций и знаки их вектора Бюргерса задаются случайным образом. Все дислокации в исходной конфигурации принадлежат к одной системе скольжения, но находятся на разных (параллельных) плоскостях скольжения, поэтому аннигиляция двух дислокаций с противоположными векторами Бюргерса в рамках данной модели исключена. Границы зерен считаются «непроницаемыми» для дислокаций (случай большеугловых границ). Если предположить, что характерное расстояние между дислокациями составляет величину порядка $10 b$, а плечо квадруполя $L$ около 600 междислокационных расстояний, то размер моделируемого зерна равен 150 нм. Тогда 50 дислокаций в расчетной ячейке приблизительно соответствуют дислокационной плотности $2.2 \times 10^{13} \mathrm{M}^{-2}$, а 500 дислокаций - дислокационной плотности $2.2 \times 10^{14} \mathrm{M}^{-2}$. В работе рассматривались дислокационные системы, содержащие от 50 до 500 краевых дислокаций.

Для оценки степени релаксации дислокационной системы рассчитывалось среднеквадратичное значение суммы диагональных компонент (шпур) тензора внутренних напряжений в зерне путем усреднения по узлам сетки, составленной из $100 \times 100$ точек. Если точки сетки находились ближе чем $0.1 b$ от ядра дислокации или дисклинации, то они в расчет не принимались.

\section{3. Результаты}

При ультразвуковом воздействии происходит значительная перестройка дислокационной структуры. Случайно распределенные дислокации двигаются по направлению к границам зерен как показано на рис. 1a и 1b. При движении они могут объединяться в триполи, изученные ранее в работах $[18,19]$, а также в более сложные мультипольные конфигурации. После ультразвукового воздействия в течение порядка 500-1000T, где Tпериод колебаний, большинство дислокаций успевают достигнуть границ зерен (см. рис. 1b). Если мощность дисклинаций в квадруполе, то есть степень неравновесности границ зерен, мала, и амплитуды внешнего ультразвукового воздействия невелики, то в границы стекают только дислокации, находящиеся в их непосредственной близости. Остальные дислокации, расположенные ближе к центру зерна, объединяясь в диполи или более сложные кластеры, остаются практически неподвижными (см. рис. 1b). Как показали исследования, такие дислокационные кластеры стабильны и после прекращения внешнего воздействия не распадаются. При повышении амплитуды ультразвукового воздействия вышеуказанных кластеров в центре зерна не образуется, и практически все дислокации оказываются в границах зерен, то есть имеет место более тщательная «очистка» зерна (см. рис. 1c). Отметим, что в обоих описанных случаях в зернах могут оставаться дислокационные диполи, которые, как было показано ранее в работах $[18,19]$, являются неподвижными при ультразвуковом воздействии. Как видно на рис. $1 \mathrm{~b}$ и 1c, из-за сил упругого отталкивания не все решеточные дислокации могут встроиться в дислокационные стенки на границе зерна, что приводит к образованию дислокационных скоплений.

На рис. 2 представлена зависимость относительного среднеквадратичного шпура тензора напряжений в каждом периоде колебаний от времени. Видно, что системы с меньшим числом дислокаций релаксируют значительно сильнее, в отличие от систем с большей дислокационной плотностью: около $22 \%$ для $n=50$, и $4 \%$ для $n=500$. Такое поведение систем с $n>200$ связано в первую очередь с образованием субструктуры, состоящей из неподвижных дислокационных стенок, диполей и мультиполей (см. рис. 1d). Отметим, что здесь для каждого $n$ мы рассмотрели только одну из многочисленных возможных конфигураций. В общем случае изменение шпура для различных систем с одним и тем же числом дислокаций различно. Проведем приблизительные оценки зависимости среднего относительного изменения среднеквадратичного шпура тензора напряжений от начального числа дислокаций системы (плотности). Для каждого $n$ рассматривалось 5 различных исходных конфигураций. Как видно из рис. 3, степень релаксации системы обратно пропорциональна числу дислокаций в ней: шпур падает в среднем на $20 \%$ для $n=50$ и на $4 \%$ для $n=500$. Отметим, что разброс значений шпура имеет тенденцию уменьшаться с увеличением дислокационной плотности.

\section{4. Обсуждение результатов}

Ультразвуковая обработка значительно ускоряет процесс перестройки дислокаций. Отдельно проведенное исследование одной и той же дислокационной структуры без ультразвука и с ним показало, что после 15000 периодов колебаний структуры значительно отличаются друг от друга. Это связано с тем, что действие периодического поля напряжений позволяет вывести из положения равновесия некоторые квазиустойчивые дислокационные кластеры, чего не происходит под действием суммарного поля напряжений дислокаций системы и дисклинационного квадруполя.

Ультразвуковая релаксация неупорядоченных дислокационных систем в конечных зернах, содержащих неравновесные границы, существенно отличается от релаксации этих систем в бесконечных кристаллах. А именно, наблюдаемое в работе [11] образование одноименных дислокационных стенок, чередующихся по знаку век- 

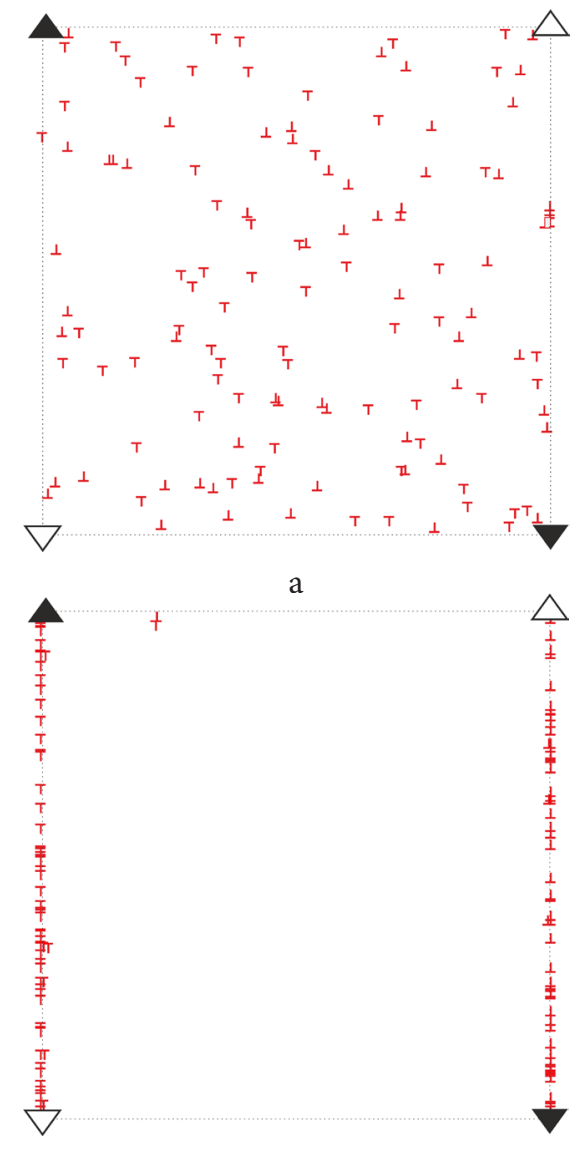

C
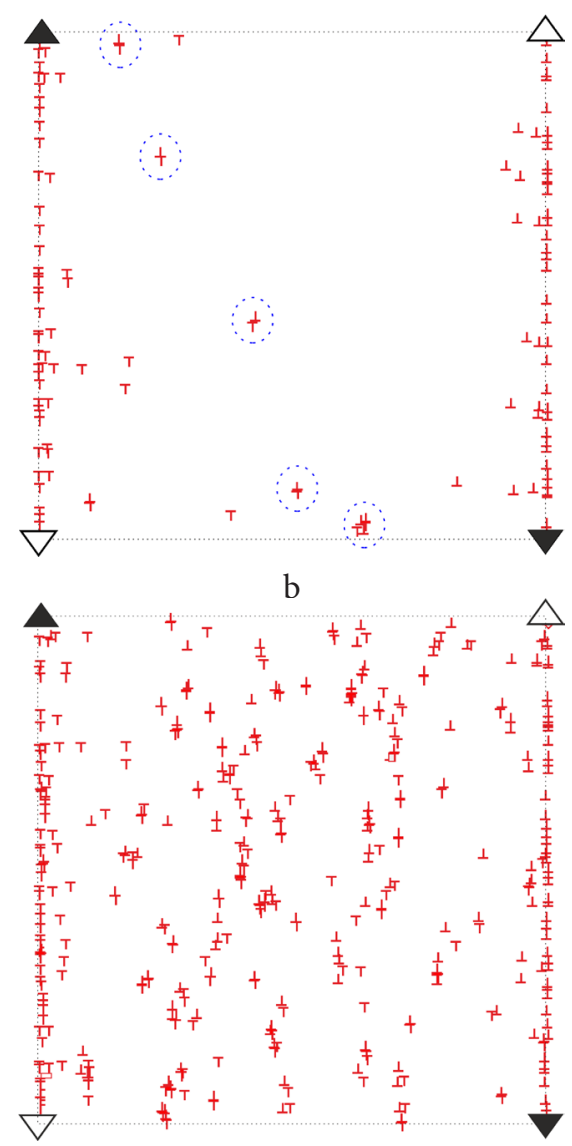

d

Рис. 1. Положения дислокаций в модельном зерне, содержащем неравновесные границы зерен: (а) исходное неупорядоченное распределение дислокаций $(n=120)$; (b) после ультразвукового воздействия при умеренной амплитуде; (c) та же структура после ультразвукового воздействия высокой амплитуды; (d) субструктура, образующаяся из неподвижных дислокационных стенок, диполей и мультиполей $(n=400)$ после ультразвуковой обработки. Черные треугольники обозначают положительную стыковую дисклинацию, а белые - отрицательную. Пунктирной линией показано квадратное зерно в модельном двумерном поликристалле. Неподвижные дислокационные диполи и более сложные мультипольные конфигурации на (b) выделены кружками.

Fig. 1. Dislocation positions in the model grain containing non-equilibrium grain boundaries: (a) initial disordered distribution of dislocations ( $n=120)$; (b) after ultrasound treatment at a moderate amplitude; (c) the same structure after ultrasound treatment at a high amplitude; (d) the substructure formed by stationary dislocation walls, dipoles and multipoles $(n=400)$. Black triangles indicate positive wedge disclination, white - negative wedge disclination. The dotted line shows the square grain in a model two-dimensional polycrystal. Stationary dislocation dipoles and more complex multipole configurations in (b) are marked with circles.

тора Бюргерса составляющих их дислокаций, не имеет места. Вместо этого дислокации из тела зерна стремятся стечь в границы зерен. Таким образом, обобщая эти два подхода, можно заключить, что в достаточно больших зернах и вдали от границ будет наблюдаться картина, описанная авторами [11], а в малых зернах и в непосредственной близости от границ дислокации будут поглощаться границами зерен.

Анализируя кривые релаксации дислокационной структуры, изображенной на рис. 2, можно выделить две стадии процесса. Первая (быстрая), соответствующая движению основной массы дислокаций в сторону границ зерен, при которой значение среднеквадратичного шпура тензора напряжений уменьшается приблизительно по линейному закону. Вторая, начинающаяся когда большинство дислокаций уже встроились в границы зерен, а оставшиеся одиночные дислокации или более сложные мультипольные конфигурации продолжают медленно дрейфовать к границам. Причем как видно из рис. 2, между этими двумя стадиями существует плавный переход и четко разделить их довольно сложно.
Существование характерного времени, при котором число дислокаций, встроившихся в стенки, не изменяется, было отмечено в работе [11] при исследовании самоорганизации дислокаций бесконечного кристалла в ультразвуковом поле. Это время насыщения составляет около 70 периодов колебаний, за которое порядка $70-80 \%$ от общего числа дислокаций всей системы встраивается в стенки. Полученная авторами [11] оценка хорошо соответствует описанной выше первой (быстрой) стадии релаксации. Таким образом, дислокационная система в конечном зерне, содержащем неравновесные границы, релаксирует в две стадии точно также как и в бесконечном кристалле, несмотря на то, что распределения дислокаций при этом значительно отличаются.

Полученные выше оценки релаксации среднеквадратичного шпура тензора напряжений от 4\% до 20\% можно сравнить с экспериментально измеренными в работе [8] значениями среднеквадратичной упругой деформации, уменьшение которой происходит из-за возврата общей дефектной структуры деформированного поли- 
кристалла, состоящей из системы стыковых дисклинаций и решеточных дислокаций. Обрабатывая ультразвуком (в широком интервале амплитуд) наноструктурный никель, полученный методом кручения под квазигидростатическим давлением, авторы [8] обнаружили снижение значений среднеквадратичной упругой деформации в интервале $10-47 \%$, а в случае ультрамелкозернистого никеля, полученного методом равноканального углового прессования, в интервале $10-16 \%$. Таким образом, име-

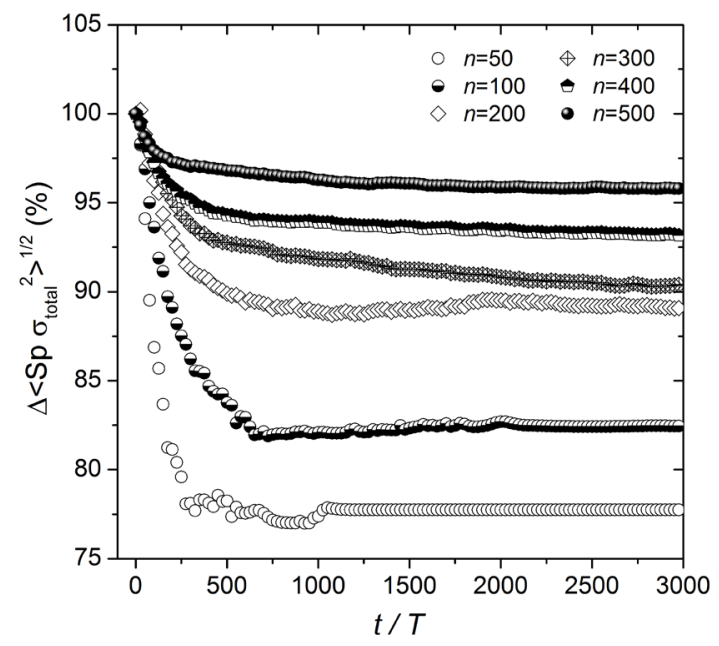

Рис. 2. Относительный среднеквадратичный шпур напряжений как функция времени (в периодах колебаний $T$ ), рассчитанный для систем с различным числом дислокаций. Значение шпура в исходной конфигурации было принято за $100 \%$.

Fig. 2. The relative root mean square trace of the stress tensor as a function of time (in oscillation periods $T$ ) calculated for systems with different number of dislocations. The value of the trace of the stress tensor in the initial configuration was taken as $100 \%$.

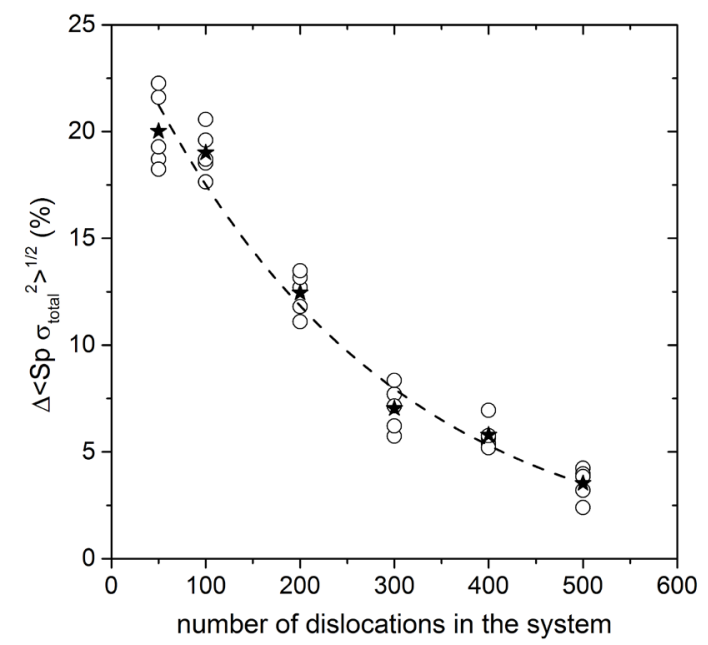

Рис. 3. Относительное изменение шпура тензора напряжений до и после ультразвукового воздействия, рассчитанное для систем с различным числом дислокаций. Значение шпура в исходной конфигурации было принято за 100\%. Средние значения шпура для каждого $n$ отмечены звездочкой.

Fig. 3. The relative change of the trace of the stress tensor before and after ultrasound treatment calculated for systems with different number of dislocations. The value of the trace of the stress tensor in the initial configuration was taken as $100 \%$. Average value of the trace for every value of $n$ is marked with asterisk. ется хорошее согласие с экспериментальными данными, даже несмотря на простоту модели, в которой рассматривались только прямолинейные дислокации, лежащие в одной плоскости скольжения, а зерно не содержало никаких точечных и линейных дефектов, которые могли бы оказывать тормозящее действие на скользящие решеточные дислокации.

\section{5. Заключение}

В рамках дискретно-дислокационного подхода было показано, что стоячие звуковые волны значительно (приблизительно в 10 раз) ускоряют перераспределение решеточных дислокаций в поле неравновесных границ зерен. Бо́льшая часть дислокаций стекает в границы зерен и выстраивается в стенку, остальные образуют в теле зерна устойчивые неподвижные конфигурации. Обнаруженные две стадии релаксации позволяют сделать предположение о том, что экспериментальное воздействие ультразвуком можно значительно сократить, что практически не должно сказаться на степени релаксации общей дефектной структуры поликристалла.

Благодарность/Acknowledgements. Настоящая работа была частично поддержана Российским фондом фундаментальных исследований (грант № 14-02-31160-мол_а). The present work was partially supported by the Russian Foundation for Basic Research (grant № 14-02-31160-мол_a).

\section{Литература/References}

1. V.P. Severdenko, V.V. Klubovich, A.V. Stepanenko. Ultrasonic pressure processing of metals. Minsk: Nauka i technika (1973) 288 p. (in Russian). [Северденко В.П., Клубович В.В., Степаненко А.В. Обработка металлов давлением с ультразвуком. Минск: Наука и техника (1973) 288 c.]

2. A.V. Kulemin. Ultrasound and diffusion in metals. Moscow: Metallurgia (1978) 200 p. (in Russian). [Кулемин А.В. Ультразвук и диффузия в металлах. М.: Металлургия (1978) 200 с.]

3. N.A. Tyapunina, E.K. Naimi, G.M. Zinenkova. Effect of ultrasound on crystals with defects. Moscow: MGU (1999) 239 p. (in Russian). [Тяпунина Н.А., Наими Е.К., Зиненкова Г.М. Действие ультразвука на кристаллы с дефектами. М.: Изд-во МГУ (1999) 239 с.]

4. O.V. Abramov, Effect of high-intensity ultrasound on liquid and solid metals. Moscow: Nauka (2000) 297 p. (in Russian). [Абрамов О.В. Воздействие мощного ультразвука на жидкие и твердые металлы. М.: Наука (2000) 297 c.]

5. I.G. Polotski, V.F. Belostotski, O.N. Kashevskaya. Phys. Chem. Mater. Treat. 4, 152-155 (1971) (in Russian). [Полоцкий И.Г., Белостоцкий В.Ф., Кашевская О.Н. Физика и химия обработки материалов. 4, 152-155 (1971)]

6. A.V. Matz, V.M. Netesov, V.I. Sokolenko, C.V. Kovtun, Problems Atomic Sci. Technol. 4, 167-169 (2009) (in Russian). [Мац А.В., Нетёсов В.М., Соколенко В.И., Ковтун К.В. Вопросы атомной науки и техники. 4, 
$167-169$ (2009)]

7. A.A. Samigullina, R.R. Mulyukov, A.A. Nazarov, A.A. Mukhametgalina, Yu.V. Tsarenko, V.V. Rubanik. Lett. Mater. 4, 52-54 (2014) (in Russian). [Самигуллина A.A., Мулюков Р.Р., Назаров А.А., Мухаметгалина А.А., Царенко Ю.В., Рубаник В.В. Письма о материалах. 4, 52-54 (2014)]

8. A.A. Nazarova, R.R. Mulyukov, V.V. Rubanik, Yu.V. Tsarenko, A.A. Nazarov, Phys. Metals Metallogr. 110(6), 574-581 (2010).

9. B.M. Loginov, A.N. Proskurin, E.V. Vershinin. Phys. Solid State. 44(10), 1799-1801 (2002) (in Russian) [Б.М. Логинов, А.Н. Проскурин, Е.В. Вершинин. Физ. Тверд. Тела. 44(10), 1799-1801 (2002)]

10. V.T. Degtyarev, A.Yu. Losev, F.A. Plotnikov. Naukoemkie tehnologii. 3-4, 5-7 (2005) (in Russian) [Дегтярев B.T., Лосев А.Ю., Плотников Ф.А. Наукоемкие технологии. 3-4, 5-7 (2005)]

11. G.V. Bushueva, G.M. Zinenkova, N.A. Tyapunina, V.T. Degtyarev, A.Yu. Losev, F.A. Plotnikov. Crystallography Rep. 53, 474-479 (2008).

12. F.A. Plotnikov, D.V. Manuhina. Vestnik TGU. 18(4), 1879-
1880 (2013). (in Russian) [Плотников Ф.А., Манухина Д.В. Вестник ТГУ. 18(4), 1879-1880 (2013)]

13. V.V. Rybin. Large plastic deformations and fracture of metals. Moscow: Metallurgiya (1986) 223 p. (in Russian) [Рыбин В.В. Большие пластические деформации и разрушение металлов. М.: Металлургия (1986) 223 с.]

14. A.A. Nazarov, R.Z. Valiev, A.E. Romanov. Scripta Materialia. 34(5), 729-734 (1996).

15. A.N. Orlov. Introduction to the theory of defects in crystals. Moscow: Vysshaya Shkola. (1983) 144 p. (in Russian). [Орлов А.Н. Введение в теорию дефектов в кристаллах. М.: Высшая Школа (1983) 144 с.]

16. J.P. Hirth, J. Lothe. Theory of dislocations. New York: Wiley (1982) 839 p.

17. V.I. Vladimirov, A.E. Romanov. Disclinations in crystals. Leningrad: Nauka (1986) 222 p. (in Russian) [Владимиров В.И., Романов А.Е. Дисклинации в кристаллах. Л: Наука (1986) 222 с.]

18. R.T. Murzaev, D.V. Bachurin, A.A. Nazarov. Phys. Metals Metallogr. 116(10), 1057-1065 (2015).

19. R.T. Murzaev, D.V. Bachurin, A.A. Nazarov. Ultrasonics. 64, 77-82 (2016). 